\title{
Report from the Participation in the Current System Workgroup
}

\author{
Nancy Davenport, Barbara DeFelice, Gary Evoniuk, Pollyanne Frantr, Julie Hannaford, \\ Jeff Mackie-Mason, Jane McAuliffe, Jennifer Pesanelli, Paul Royster, Crispin Taylor, Michael Wolfe
}

\section{OSI2016 Workgroup Question}

Do researchers and scientists participate in the current system of scholarly publishing because they like it, they need it, they don't have a choice in the matter, or they don't really care one way or another? What perceptions, considerations and incentives do academicians have for staying the course (like impact factors and tenure points), and what are their pressures and incentives for changing direction (like lowering publishing charges)?

\section{Premise and objectives}

The authors of scholarly works play a critical role in the scholarly communications system: authors are the original content creators, and in many or most cases are the original rightsholders and the ultimate decisionmakers when it comes to how, when, and where to publish their work. Although there are other significant participants in the current system (including publishers, librarians, information consumers, etc.), understanding and respecting the range of influences that shape author publication decisions are crucial to effecting change in the system.

While recognizing the highly individual and diverse nature of author interests, we identified several priorities that stand out as driving decisions in the publication process. Career advancement concerns are primary, and the perceived currency of a publication mode or venue with promotion and tenure committees is a significant factor in decision making. A related, but distinct, factor is a publication venue's perceived prestige among the authors' peers. Both of these considerations have significant interplay with, and often serve as proxies for, scholarly authors' overarching motivation to advance knowledge and make an impact in their fields. External factors may also direct author choice. Funder requirements and, in the case of works made for hire, employer requirements, can narrow the range of options available to authors.

Survey evidence suggests that authors increasingly see open publication models as being consistent with, or in furtherance of, their goals as scholars. ${ }^{1}$ For instance, authors increasingly see open access (OA) publication as leading to wider circulation, greater visibility, and possibly more citations. Our task was to consider how we might accelerate these trends to facilitate openness in scholarship.

Our workgroup considered how to build an author-focused model of change toward 
OA. First, taking into account the motivations highlighted above, we characterize how the system might be built to address authors' interests-what an author's perfect world of open scholarship might look like. We then turn to obstacles, both cultural and structural, that might inhibit this kind of change. Finally, we issue a series of proposals to help bring about author-centered change toward more open scholarly communications practices, and identify areas where further research is needed.

\section{The author's perfect world}

To guide our assessment of challenges and develop our proposal for increasing participation in the open system, we characterize a perfect open scholarship world for authors. Although such an idealization itself will be imperfect, it does provide a starting point for identifying obstacles and proposing solutions.

First and foremost, authors would have clear, expansive, and persistent rights. They would not have to be experts in copyright law or understand the nuances differentiating the growing array of "open" licenses in order to decide what type of license to apply to their work. Licensing terms would be flexible enough to allow authors to meet the needs of their institutions and funders as well as their own needs to use and reuse their work over time. In addition, their rights, established at the time of publication, would persist regardless of changes such as a publisher changing policies or platform migrations or upgrades.

From the author viewpoint alone, publication in a perfect world would be free. Of course, publication requires human effort and other resources, and so solely from a social welfare perspective, only those publications with expected social value greater than the underlying resource cost of publication would be published. However, since the benefits largely accrue to society beyond the author, those costs should, in general terms, be borne socially, not by the authors. (Those costs might include, for example, provision of some incentives to authors to participate effectively in the open scholarship system.) We recognize that there may be some personal benefits to publication, and so it may not be social welfare-decreasing for authors to bear a small portion of the cost of publication, so long as it is small enough as to not interfere with the decision to publish scholarship for which the social benefits are larger than the resource cost of publication.

In terms of a thriving monograph environment, authors would have options for long-form publication that permit OA licensing, are affordable, and sustain the quality-assurance measures appropriate for the field and format. Anecdotal evidence from recent publishing markets suggests that open online publication is not inconsistent with sales of physical books. A number of open-access monograph publishing outlets already exist, and several cooperative projects (by the Public Knowledge Project and the Oberlin Group) are currently underway or in planning stages. Other projects (such as Knowledge Unlatched and Luminos) seek to fund open licenses for monographs by assembling pre-publication financial support from prospective users. Whatever the methods or funding models, authors need venues that are affordable or free, or they need institutional support to remunerate publishers willing to risk loss of income from licensing fees. One important development will be the evolution of tiered levels of service, rather than one-size-fitsall packaging for editorial development, production, marketing, and dissemination. 
Ideally, authors will be able to select from among a range of venues, services, and pricing. An essential element will be the support of institutions and societies in establishing or supporting open imprints for monographs that are attractive in terms of quality and reputation.

Ideally authors will face low administrative burdens for publishing in open channelsat least as low as for publishing in closed channels. Submission systems will be streamlined and format neutral. Delays from the publishing process will be small.

Finally, in the author's perfect open scholarship world, there will be more and better metrics for assessing the impact of scholarly work. The resulting data will be provided to authors in a timely, meaningful manner, and the metrics will be readily accessible to institutions and all end-users as well.

If this were the world of open scholarship and publication, authors would participate. What are the barriers to helping it to occur?

\section{Inhibitors and challenges}

Amongst authors, a considerable spectrum of attitudes exists toward a more open scholarly publishing environment. For those not presently participating in open, these range from simple apathy to outright opposition. Authors face a range of inhibitors that limit their willingness to engage in OA practices. They include the following:

- In some disciplines, there is a strong perception that everyone who "needs" to read their scholarship already can. Scholarly networks are small and closed, with proven mechanisms for informal communication. Additionally, libraries provide seamless access to the published record. Many scholars are unaware of or unconcerned about: a) the high costs associated with journal subscriptions, and b) the audience restrictions that licenses place on access to journal content. As a result, many fail to consider that those beyond the academy can neither access nor read their work.

- Participating in a more open manner can take extra time, which for many academics is one of the most precious resources. This is particularly the case for "green" OA, or the self-archiving of journal articles to institutional or subject repositories. Many are convinced of the merits of self-archiving, but perceive the work involved to be too onerous and therefore fail to make it a priority.

- Many authors have a vested interest in the status quo. They may feel loyalty to their discipline's scholarly society and perceive a move towards greater openness as a threat to the society journals' ongoing viability, which, to date, have been heavily reliant on subscriptions. Additionally, those who are successful in the current system are likely motivated to preserve their existing level of prestige. Herb (2010) describes the scientific capital authors derive from current practices; this becomes a powerful driver to defend existing approaches to scholarly publishing. ${ }^{2}$

- Across disciplines, authors receive varying amounts of funding support, particularly for publishing fees. Humanities scholars are less likely to be recipients of grant funds, compared to their colleagues in the sciences. Article processing charges (APCs) themselves can range from a few hundred to thousands of dollars. Some, but not all, 
institutions have made funds available for authors to apply for APC support.

- Many authors lack clarity regarding their rights related to $\mathrm{OA}$ publication. Publishers' copyright policies are often arcane, contradictory, and subject to frequent, unannounced change. Authors are therefore understandably confused about what they are permitted to do with their own work and are reluctant to take actions that might put them at odds with their publisher. In many cases, scholars are unaware that they can negotiate to keep their own copyright, therefore losing the opportunity to have greater control over their own research outputs.

- Many authors fear loss of a competitive edge if they publish their research in OA venues. They anticipate being "scooped" or having their work compromised via plagiarism. Given the fact that open publishing provides an unmistakable record of their work, these concerns seem counterintuitive; however, they are firmly entrenched in many disciplines.

- Many authors believe that they have complied with funding requirements for OA, but the publisher itself fails to comply. For example, the Wellcome Trust (2016) has found Elsevier and Wiley to be particularly problematic in terms of complying with the requirement to archive journal articles in PubMed Central. ${ }^{3}$

\section{Proposals}

Overcoming these obstacles and building an authoring environment more conducive to open will require significant cultural, institutional, and structural changes, as described below.

\section{Cultural change}

A key force in motivating change in the level of author participation in open dissemination is the academic and research culture itself. So it will be necessary to work within this culture to support change, as well as building robust structures to enable that change, as we detail below in our proposal. As early career researchers and scholars understand, practice, and even advocate for options that make their work more open, and at the same time succeed in the current system, that system itself will gradually change. On the other end of the career path, well established scholars and scientists speaking out about and practicing open dissemination will provide crucial role models for early career researchers and scholars. To enable this cultural change, working within and across research and academic institutions, programs need to be created that provide:

- exposure to the wide variety of options to make work open in the scholarly publishing and dissemination environment;

- awareness of ways that this direction is supported through funding, from the institutional level, the scholarly and scientific society level, national level and even global level;

- awareness of the requirements for sharing the results of funded research; and

- awareness of the real benefits of doing work in the open.

\section{Consistent messaging}

A collective effort is also required to develop a concise and consistent message about open scholarship given the widespread misconceptions, confusion and even fear about OA. A unified message 
that both informs and involves stakeholders is an important step toward accelerating cultural change within the current scholarly publishing system. International stakeholders from the following groups should be invited to develop the message:

- faculty researchers and scholars (pretenure and tenured)

- student researchers (undergraduate, graduate and postdoctoral)

- academic administrators

- faculty governance groups (senate and tenure and promotion committees)

- government, foundation, and industry funders

- publishers (commercial, university presses, scholarly societies)

- librarians

- scholarly and professional societies

- complementary organizations (Federal Demonstration Partnership, Government-University-Industry Research Roundtable, Authors Alliance, etc.).

This unified message should address the inhibitors and challenges through a positive, action-oriented focus. It should define the issue; emphasize the positive aspects of embracing open scholarship; convey that authors have a choice whether to support open scholarship; and be adaptable to being delivered to different stakeholder groups.

In particular, attention must be paid to dispel the notion that open scholarship has little to no positive impact. Once this is addressed, reservations by tenure and promotion committees and senior academic administrators may begin to fade.

\section{More high-value open communica- tion vehicles}

In order to overcome apathy and resistance, we need more and better venues for open publication, regardless of field, specialization, or medium. For most authors, editorial and reputational quality will continue to be the primary considerations, but open venues can increase participation by providing more value added. For instance, such open vehicles might provide:

- Clear, expansive, and persistent author rights. Open venues, being by their nature less dependent on exclusivity, are best positioned to provide authors expansive and persistent reuse rights, currently lacking in other fora.

- Author-focused impact metrics. The availability of detailed, up-to-date, and cutting-edge metrics will help assure authors that they can demonstrate their work's impact without relying on journal-level metrics.

- Valuable content enhancements. Particularly relevant to the open effort would be those enhancements that serve to make work more discoverable on the open web.

- Content promotion. Enhanced visibility would complement the added accessibility promised by open models.

\section{Institutional commitments to schol- arly communication efforts}

The greater social value of open scholarship justifies additional resource commitments by universities, funding agencies, and other institutional stakeholders. For example, in universities some inducements that might increase author participation include:

- Creating and adjusting author incentives. Universities might reward 
authors for publishing in low-cost OA venues (for instance, by adding funds thus saved from publishing in highercost venues to the author's research budget), or develop policies to better credit demonstrated commitments to open access in the promotion and tenure process. Likewise scholarly and scientific communities could recognize and reward open practices and behaviors.

- Reducing friction in open practices. Both green and gold approaches to $\mathrm{OA}$ can add extra steps to the publication process for authors. Institutions should commit resources to reducing these sources of friction wherever possible-for instance by assisting with submission to institutional repositories and reducing administrative burdens associated with securing and paying for gold open access.

- Supporting open venues. Creating more high-value open communication vehicles, as noted above, will require stakeholder institutions to redirect expenditures toward open, whether in the form of diverting subscription budgets toward processing charges or in providing direct financial support to promising new venues and models.

- Providing scholarly communication education and support. Discussing the benefits of open practices in earlycareer trainings as well as providing meaningful support on scholarly communication are key components of building the consistent messaging discussed above.

These investments are likely to pay off in the future through greater awareness of the options for publishing work, more high value choices for authors, and lower overall costs of publishing.

\section{Research agenda}

There are two kinds of research we believe could be beneficial in solidifying reform efforts. First, it would be helpful to disentangle the many factors that influence author decisions, in order to illuminate those areas where reforms would be most effective. For instance, how do publishing behaviors change (if at all) before and after tenure or other career milestones? The nature of the change would be helpful in gauging the role of promotion and tenure in decisionmaking toward publishing in open venues.

Second, further research into the practical effects of open behaviors could better make the case to authors that open practices can provide professional rewards. We welcome further research on the impact of open on citations, but also into its effects on press coverage, innovation, social advancement, policy, and inclusivity. In the monograph space, where authors might hope to earn royalties on their publications, further research into the impacts of open access on print sales and revenue could be important both to skeptical authors and to open-oriented authors looking to persuade presses of the viability of open publications.

\section{Conclusion}

We are not yet in a "perfect world" for open publishing from the scholarly author's perspective. Despite disparate author motivations and some measure of enduring skepticism toward open models, however, we have identified proposals for reforms, messaging, and research that could address many common author concerns and create a more hospitable framework for authors to participate in the open publishing system. 


\section{OSI2016 Participation Workgroup}

Nancy Davenport, University Librarian, American University

Barbara DeFelice, Program Director for Scholarly Communication, Copyright and Publishing, Dartmouth College

Gary Evoniuk, Director of Publication Practices, GlaxoSmithKline (GSK)

Pollyanne Frantz, Executive Director, Grants Resource Center, American Association of State Colleges and Universities (AASCU)

Julie Hannaford, Deputy Chief Librarian, University of Toronto

Jeff Mackie-Mason, Dean of Libraries, University of California Berkeley

Jane McAuliffe, Director, National and International Outreach, Library of Congress

Jennifer Pesanelli, Deputy Executive Director for Operations and Director of

Publications, Federation of American Societies for Experimental Biology (FASEB)

Paul Royster, Coordinator of Scholarly Communications, University of NebraskaLincoln Libraries

Crispin Taylor, Executive Director, American Society of Plant Biologists

Michael Wolfe, Executive Director, Authors Alliance

\section{Notes:}

\footnotetext{
${ }^{1}$ Frass, W., Cross, J., \& Gardner, V. “Taylor \& Francis Open Access Survey,” Taylor \& Francis, June 2014, as of June 13, 2016: http://www.tandf.co.uk/journals/explore/open-access-survey-june2014.pdf.

${ }^{2}$ Herb, Ulrich, "Sociological implications of scientific publishing: Open access, science, society, democracy, and the digital divide," First Monday, 15(2), February 2010, as of June 13, 2016: http:// firstmonday.org/ojs/index.php/fm/article/view/2599/2404.

${ }^{3}$ Wellcome Trust, "Wellcome Trust and COAF open access spend, 2014-15," March 23, 2016 as of June 13, 2016: https://blog.wellcome.ac.uk/2016/03/23/wellcome-trust-andcoaf-open-access-spend-2014-15/.
} 